\title{
Response to Letter to the Editor: Actioning our understanding of respiratory compromise
}

\author{
Justin Sorge, RRT FCSRT MPH, Editor-in-Chief
}

J Sorge. Response to Letter to the Editor: Actioning our understanding of respiratory compromise. Can J Respir Ther 2019;55:30. doi: 10.29390/cjrt-2019-001.

In their letter to the editor, appropriately titled "Actioning our Understanding of Respiratory Compromise," Corriea, Wnuk, Zaccagnini, and West [1] rightly describe the difficulties in developing a case definition of Respiratory Compromise (RC). The authors go on to detail some of the national efforts of the Canadian Society of Respiratory Therapists and its Respiratory Compromise Advisory Workgroup. These efforts are commendable and I applaud the work carried out by this group. This letter also presents an opportune time to identify a potential source of confusion concerning the case definition of RC.

The authors refer to studies conducted on the Get with the Guidelines Resuscitation (GWTG-R) data registry [2]. This registry uses a case definition of RC quite different than that put forth by Morris et al. [3], who define RC as "...a deterioration in respiratory function in which there is a high likelihood of decompensation into respiratory failure or death but for which timely intervention...might prevent or mitigate decompensation". In contrast, the GWTG-R data registry defines RC as "absent, agonal or inadequate respiration that requires emergency assisted ventilation, including non-invasive...or invasive... positive pressure ventilation" $[2,4,5]$.

It is clear that these definitions describe two related, although not equal, populations. It is safe to say those meeting the GWTG-R definition will also meet the definition provided by Morris. But it would be incorrect to say the reverse is true, that all cases determined to be in $\mathrm{RC}$ by the Morris definition would remain classified as such by the GWTG-R definition. That is, an undetermined proportion of Morris-defined cases would not deteriorate to a state requiring positive pressure ventilation (PPV).

But does this matter? I suggest the differences do when attempting to describe the epidemiology of RC. For instance, Corriea and colleagues [1] state that $90 \%$ of cases of RC require PPV or endotracheal intubation, which is true under the GWTG-R definition of RC. However, this proportion would likely be lower had the denominator (i.e., cases of RC that do not lead to PPV or intubation) included additional cases as defined by the Morris criteria. The problem can be illustrated quantitatively using some dummy data:

Let $A_{1}=$ cases of RC that require PPV or endotracheal intubation, assume 90 ,

$A_{0}=R C$ cases as defined by the GWTG-R, assume 100,

$P_{\text {GWTG-R }}=$ prevalence of cases of RC that require PPV or endotracheal intubation using the GWTG-R definition, assume 0.90 (i.e., 90\%),

$B_{0}=R C$ cases as defined by the Morris definition but do not meet the GWTG-R definition, assume 100 (i.e., a proportion of RC cases meeting the Morris definition equal to that identified by the GWTG-R definition would not progress to requiring $\mathrm{RC}$ or intubation),

$P_{\text {Morris }}=$ prevalence of cases of RC that require PPV or endotracheal intubation using the Morris definition (unknown).
Using this information, $P_{\text {GWTG-R }}$ can be calculated:

$$
P_{\text {GWTG-R }}=\frac{A_{1}}{A_{0}}=\frac{90}{100}=0.90
$$

However, calculating $P_{\text {Morris }}$ reveals the effect of the inflated denominator, using the less specific Morris definition relative to the GWTG-R definition:

$$
P_{\text {Morris }}=\frac{A_{1}}{A_{0}+B_{0}}=\frac{90}{100+100}=0.45
$$

So, it would be incorrect to say that the prevalence of cases of $\mathrm{RC}$ that require PPV or endotracheal intubation using the Morris definition is also $90 \%$. The example above, assuming an arbitrary but greater number of RC cases using the Morris definition, shows that this prevalence would in fact be smaller! This would follow through to the other statistics presented by Corriea and coauthors [1]. This example shows how differences in representativeness of data derived from two different, but related, case definitions may lead to divergent results.

I agree with the authors of the letter to the editor that settling on a standardized case definition of RC will be difficult. I also agree that the "perfect definition" may not be achievable. But I believe that it is a good end-point to strive towards. Perhaps moving forward, it would be prudent to identify from which populations or definitions our statistics arise in discussions concerning $\mathrm{RC}$ to ensure we are comparing like with like; especially important while these discussions are emergent. Because sometimes, it seems, RC by another definition smells different.

\section{REFERENCES}

1. Correia R, Wnuk A, Zaccagnini M, West A. Actioning our understanding of respiratory compromise. Canadian Journal of Respiratory Therapy. 2019;55:28-29.

2. Peberdy MA, Kaye W, Ornato JP, et al. Cardiopulmonary resuscitation of adults in the hospital: A report of 14720 cardiac arrests from the National Registry of Cardiopulmonary Resuscitation. Resuscitation 2003;58(3):297-308.

3. Morris TA, Gay PC, MacIntyre NR, et al. Respiratory compromise as a new paradigm for the care of vulnerable hospitalized patients. Respiratory Care 2017;62(4):497-512.

4. Andersen LW, Berg KM, Chase M, Cocchi MN, Massaro J, Donnino MW. Acute respiratory compromise on inpatient wards in the United States: Incidence, outcomes, and factors associated with in-hospital mortality. Resuscitation 2016;105:123-9.

5. Moskowitz A, Andersen LW, Karlsson M, et al. Predicting in-hospital mortality for initial survivors of acute respiratory compromise (ARC) events: Development and validation of the ARC score. Resuscitation 2017;115:1-10.

Justin Sorge, Research Associate, Canadian Institute of Substance Use Research, University of Victoria, Victoria, BC

Correspondence: Justin Sorge. Email: editorinchief@csrt.com

Published online at https://www.cjrt.ca on 15 February 2019 